\title{
Simulation of Excited Molecule Propagation to Determine Plasma Sterilization Effectiveness in Lumens
}

\author{
Clair Koo' ${ }^{1}$ Andy Poggio", and Tarn Wilson" \\ ${ }^{1}$ Henry M. Gunn High School, Palo Alto, CA, USA \\ \#Advisor
}

$\underline{\text { ABSTRACT }}$

Sterilizing or disinfecting medical narrow-lumen instruments such as endoscopes is a highly difficult process. Although plasma sterilization is effective against a broad spectrum of microorganisms, its effectiveness is dependent on the density of plasma-generated, excited particles that attack the microorganisms. The lifetime of the excited particles is not long, and their survival is a function of operating condition and geometry. The current study employs a numerical method to investigate the propagation and density of the excited nitrogen molecules in a narrow lumen and to identify the conditions where the plasma sterilization is effective. The plasma gas properties are calculated using the gas kinetic theory, and different lifetimes are assumed for excited molecules. The simulation results show that the survival of excited particles in a lumen increases more than 10 times when the pressure is reduced by a factor of 10 . Thus, it is highly recommended that the plasma be operated at lower pressure to increase the sterilization efficiency of narrow lumen devices.

\section{Introduction}

\section{Sterilization of Lumen Medical Devices}

The sterilization of medical devices before use is critical in preventing secondary infections. The devices are sterilized in a variety of ways: steam, dry heat, radiation, and chemical gases such as ethylene oxide, hydrogen peroxide, formaldehyde, and peracetic acid (Sakudo et al., 2019). Despite the multiple methods of sterilization, the sterilization or disinfection of narrow-lumened instruments, such as endoscopes, is still a very challenging process (Sanchez, 2019).

Narrow-lumened medical devices are characterized by their long and thin channel configurations. They are important tools for the diagnosis and treatment of a wide variety of health problems (Prust, 2018). Endoscopes are one of the most well-known narrow-lumen devices, are used to observe the digestive system through natural openings of the body, and have an instrument channel width of about $2 \sim 2.8 \mathrm{~mm}$ (Kay and Wyllie, 2013). They are sophisticated devices constructed with multiple specialized materials and parts, including optics, electronics, and multiple polymers (Prust, 2018). As these devices are expensive, health care facilities try to minimize the reprocessing time and optimize their use (Prust, 2018).

Multiple factors contribute to the complexity of sterilizing lumens. They are from $300 \mathrm{~mm}$ to $1.7 \mathrm{~m}$ in length, too long to perform effective low-temperature chemical sterilization due to the limited delivery of the sterilant and the risk of residuals (Sanchez, 2019). Low-temperature sterilization using chemicals, such as ethylene oxide, requires long exposure and aeration periods that can limit the availability of the devices and risks of leaving hazardous residue (Sanchez, 2019). Furthermore, the materials are incompatible with sterilization methods involving high temperatures 
(Sanchez, 2019). Plasma sterilization is a promising technology that enables fast low-temperature processing without any chemical residues (Sakudo et al., 2019).

\section{Plasma Sterilization}

Plasma is the fourth state of matter in addition to solid, liquid, and gas (Nandkumar, 2014). As energy is applied, a solid becomes a liquid and a liquid becomes a gas. When the amount of applied energy reaches a point where the gas molecules become energized ions and electrons, plasma is generated (Nandkumar, 2014). Plasma, generally defined as an ionized, energized gas containing balanced charges of ions and electrons, is composed of various excited species, including atoms, molecules, electrons, ions, free radicals, and photons (Fridman, 2008). Plasma is achieved through an ionization process in which electrons or photons carrying sufficient energy collide with neutral atoms or molecules (Silva et al., 2010). The resulting plasma contains reactive, excited chemical species and emits visible, ultraviolet (UV), and infrared radiation (Fridman, 2008). Due to its unique state, plasma has many important applications, such as semiconductor device fabrication, surface treatment, and waste treatment (Fridman, 2008).

Various types of plasma can be classified according to their temperature and equilibrium state (Fridman, 2008). In a thermal plasma (hot plasma), the temperatures of the electrons and excited heavy particles such as ions and neutral molecules are very close or the same, and the heavy particles are in thermal equilibrium through frequent collisions (Fridman, 2008). In non-thermal plasma (cold plasma), the temperature of the electrons is significantly higher than that of the excited heavy particles, and gas temperature remains near the ambient temperature (Fridman, 2008). Non-thermal plasma is generally produced under low-pressure conditions (Fridman, 2008). At low pressure, collisions between particles (electrons, ions, and neutral molecules) occur less frequently, and the excited particles can travel for longer without losing energy through collisions. Subsequently, the lifetime of excited particles is longer at a lower pressure. Since the larger excited particles are at or near ambient temperatures and the electrons at a high temperature have relatively few collisions with excited particles, the overall plasma gas remains at a low temperature (Shintani et al., 2010). As a result, non-thermal plasma is most suitable for biological applications such as sterilization (Sakudo et al., 2019).

In recent decades, research has focused on the plasma sterilization and disinfection of medical devices (Stapelmann, 2013). Plasma can inactivate microorganisms with high levels of resistance, including infectious prions (Sakudo et al., 2019). However, the inactivation mechanisms of microorganisms by plasma are still not clearly understood (Sakudo et al., 2019; Silva et al., 2010; Shintani et al., 2010). Mechanisms depend on the plasma gas, the plasma generation method, and the target microorganisms. In the case of nitrogen gas plasma, major inactivation mechanisms are thought to be UV radiation and reactive species eroding microorganisms directly (Shintani et al., 2010). In the case of oxygen plasmas, etching, a mechanism that removes material from the surface chemically, is also a major factor in the inactivation process (Traba, 2013). Further studies are required to understand the plasma inactivation mechanisms of various microorganisms.

\section{Plasma Sterilization of Narrow-Lumen Medical Devices}

The use of inert gas plasma, such as nitrogen and argon plasma, can achieve the required sterilization process, as well as meet the material compatibility (Shintani et al., 2010). It is important to meet both effectiveness and compatibility during sterilization applications. However, some inactivation mechanisms are not effective in all conditions. Geometric restrictions, such as complicated configurations and narrow tubes, limit the direct exposure of the inner surfaces to UV radiation sterilization. The delicate materials of lumen devices may not be compatible with the oxygen plasma with the etching effect. The major inactivation mechanism of inert gas plasma is the erosion of microorganisms through the direct attack of excited molecules without chemical etching. Thus, it is a potential solution for the sterilization of delicate narrow lumen medical devices. 
The availability or density of excited (energized or activated) inert gas molecules is the key parameter in inactivating the microorganisms. However, the lifetime of excited particles is limited and is a function of operating conditions, such as temperature, pressure, and space configuration (Fridman, 2008). An excited inert gas molecule loses energy during collisions with other molecules and eventually becomes a ground-state molecule when it loses all of its excited energy. In a confined geometry, such as a narrow lumen, its lifetime is shorter due to additional wall collisions.

The current study investigates the propagation and survival of the excited nitrogen molecules in a narrow lumen geometry filled with nitrogen at a low-pressure and identifies the conditions where plasma sterilization is effective. The nitrogen molecules are energized and released at the opening of the lumen device. Assuming that the major inactivation mechanism is the erosion of the microorganism by the attack of excited molecules, the density or number of excited molecules near the microorganism target determines the effectiveness of the plasma sterilization process. In the current theoretical (and simulation) approach, the molecules' properties are approximated using the gas kinetic theory, and their movement is tracked using the Monte Carlo Method.

\section{Gas Kinetic Theory}

Tracking molecular movement requires information about each molecule's kinetic properties, including diameter, mass, velocity, collision frequency, and distance between collisions. This study employs the gas kinetic theory to identify or estimate these properties. Gas kinetic theory defines gas as a group of very small, individual molecules in constant motion. Although there is no gas movement at a macroscopic level, each molecule experiences random motion and consecutive collisions with other molecules and any surfaces that are present. Gas kinetic theory relates the macroscopic properties of gas, such as temperature and pressure, to the microscopic characteristics of each molecule including mass, size, and motion (Vincenti and Kruger, 1975).

Among various molecular models in the gas kinetic theory, the current study employs the simplest 'billiardball' model where the gas molecules are rigid, perfectly elastic spheres with no attractive intermolecular forces and with repulsive force only at contact with other molecules (Vincenti and Kruger, 1975). Each gas molecule is given mass $m$ and diameter $d$ depending on the types of gases. The diameter of a molecule in the billiard-ball model is the scattering diameter (or kinetic diameter), determined by intermolecular collision events (Vincenti and Kruger, 1975). The gas species of interest in this study is nitrogen $\left(\mathrm{N}_{2}\right)$. Its molecular weight $M$, molecular mass $m$, and scattering diameter $d$ of are as follows (Ismail et al., 2015).

$$
\begin{aligned}
& \text { Molecular weight: } M=28.01 \mathrm{~kg} / \mathrm{kmol} \\
& \text { Molecular mass: } m=M / N_{A}=4.65 \times 10^{-26} \mathrm{~kg} \\
& \text { Scattering diameter: } d=3.64 \times 10^{-10} \mathrm{~m}
\end{aligned}
$$

where $N_{A}$ is Avogadro's number $\left(6.022 \times 10^{26} \mathrm{kmol}^{-1}\right)$, representing the number of particles per kmole. The number density $n$, defined as the number of molecules in a unit volume, is also a key property to simulate the interaction between molecules and can be calculated from the ideal gas law.

$$
\text { Number density: } n=P /\left(k_{B} \cdot T\right)
$$

where $P$ is the pressure in $\mathrm{Pa}, k_{B}$ is Boltzmann constant $\left(k_{B}=1.381 \times 10^{-23} \mathrm{~J} / \mathrm{K}\right)$, and $T$ is the gas temperature in $\mathrm{K}$. The Boltzmann constant is the gas constant for a molecule and can be calculated as $k_{B}=R / N_{A}$ where $R$ is the universal gas constant $(8.314 \mathrm{~kJ} / \mathrm{kmol}-\mathrm{K})$. At $1 \mathrm{~atm}(101,325 \mathrm{~Pa})$ and $0^{\circ} \mathrm{C}(273.15 \mathrm{~K})$, the number density of molecules is calculated as $2.687 \times 10^{25} \mathrm{~m}^{-3}$.

The current study investigates the movement of excited $\mathrm{N}_{2}$ molecules in a narrow lumen device filled with nitrogen. When the excited $\mathrm{N}_{2}$ molecules enter the device, they collide with the neighboring $\mathrm{N}_{2}$ molecules. Tracking 
each excited molecule requires kinetic information about velocity and distance between collisions. The current study assumes every molecule moves at the average velocity and travels the average distance between collisions. From the equipartition theorem of the gas kinetic theory, the average velocity $U$ of molecules can be estimated as (Vincenti and Kruger, 1975).

Average molecule velocity: $U=\sqrt{3 k_{B} T / m}$.

The mean free path $M F P$, defined as the average distance that a molecule travels between successive collisions, can be calculated using Equation (3) below (Vincenti and Kruger, 1975). The frequency of collision and collision interval can be calculated using the average velocity and mean free path.

Mean free path: $M F P=1 /\left(\sqrt{2} \pi d^{2} n\right)$

Collision frequency: $f=U / M F P$

Collision interval: $t_{s}=M F P / U$

\section{Monte Carlo Method}

To study the propagation of excited $\mathrm{N}_{2}$ molecules in a lumen device, the current study uses the Monte Carlo method (or Monte Carlo simulation), which considers the microscopic random behavior of their movements and collisions. The Monte Carlo method is a computational algorithm utilizing repeated random sampling and statistical analysis (Harrison, 2010). It has been widely employed in many areas including economic, mathematical, medical, and physical problems (Harrison, 2010). When it is difficult to use other numerical analysis approaches, this method is especially useful for simulating physical phenomena related to fluid mixing, Brownian motion, interacting particles, etc. (Brownlee, 2019).

Each excited molecule's collision and movement is tracked to determine the population of excited molecules in terms of time and position. The code programmed in Python performs the physics calculation that follows each molecule's movement and position. Figure 1 shows the geometry under consideration. For simplicity, the lumen is modeled as a long tube with a square cross-section with $2 \mathrm{~mm}$ width according to the typical endoscope width (Kay and Wyllie, 2013).

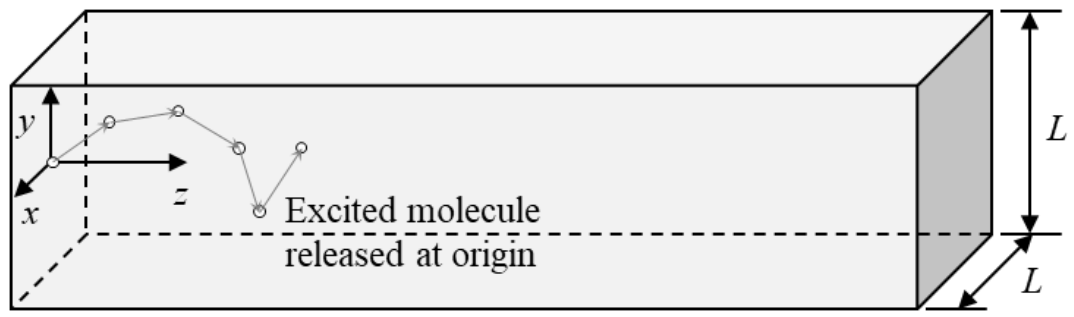

Figure 1. Simplified geometry for a narrow-lumen device

Initially (at time $t=0$ ), excited $\mathrm{N}_{2}$ molecules are released at the origin $(0,0,0)$ in the opening to simulate the propagation of plasma gas injected into the device. The calculation follows each molecule's movement and position at each collision. The molecule's moving direction is determined by random numbers generated by the Python code. After traveling the mean free path, the molecule collides with another $\mathrm{N}_{2}$ molecule and changes its direction which is determined by another set of new random numbers generated by the program. This process repeats as the molecule travels the mean free path to collide with another $\mathrm{N}_{2}$ molecule. At each collision, the particle's location is calculated 
by adding the displacement to the previous collision location. It is assumed that the collision has a diffusive effect on the direction, meaning that the new direction has a uniform probability in all angles after each collision. Figure 2 describes the molecular collision and two random angles $\alpha$ (polar angle, $0 \leq \alpha \leq \pi$ ) and $\beta$ (azimuthal angle, $0 \leq \beta \leq$ $2 \pi$ ) to determine the new moving direction in the following equations.

Velocity in z direction: $U_{z}=\mathrm{U} \cdot \cos \alpha$

Velocity in x direction: $U_{x}=U \cdot \sin \alpha \cdot \cos \beta$

Velocity in y direction: $U_{y}=U \cdot \sin \alpha \cdot \sin \beta$

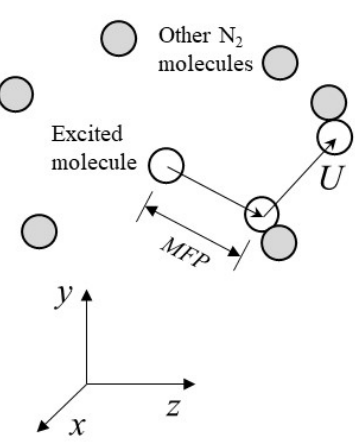

(a)
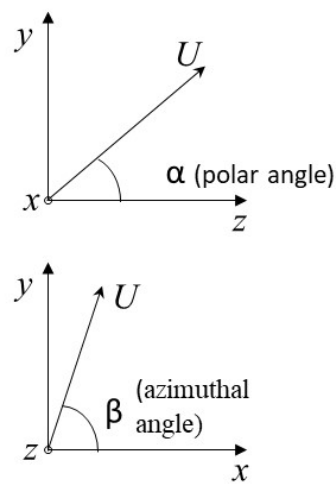

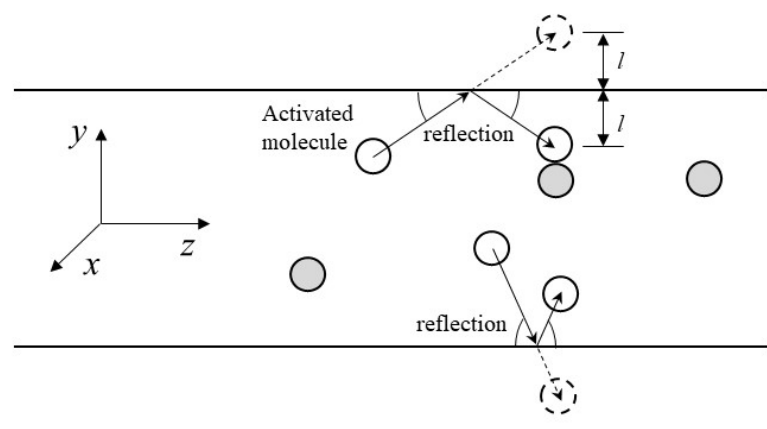

(b)

Figure 2. Molecule's movement and collision: (a) intermolecular collision and movement and (b) reflection and bouncing of molecules on the wall.

The molecules also collide with the inner wall of the device. As the wall is fixed, the current study assumes that the molecule's movement after molecule-wall collisions is directional and reflective relative to the wall. The movement angle after each collision is calculated shown in Figure 2(b).

Python's random number generation function "random.uniform" is used to generate these angles with uniform distribution.

Polar angle: $\alpha=$ random.uniform $(0,1) \times \pi$

Azimuthal angle: $\beta=$ random.uniform $(0,1) \times 2 \pi$

Figure 3. is the histogram that shows the distribution of random numbers generated by "random.uniform $(0,1)$ " function of Python. 10,000 random numbers were generated, and the bin size of the histogram is 0.1 . The average and standard deviation of the bin population are $10 \%$ and $0.26 \%$, respectively. It indicates that the random sampling of numbers between 0 and 1 is very uniform and can be applied to the current study. 


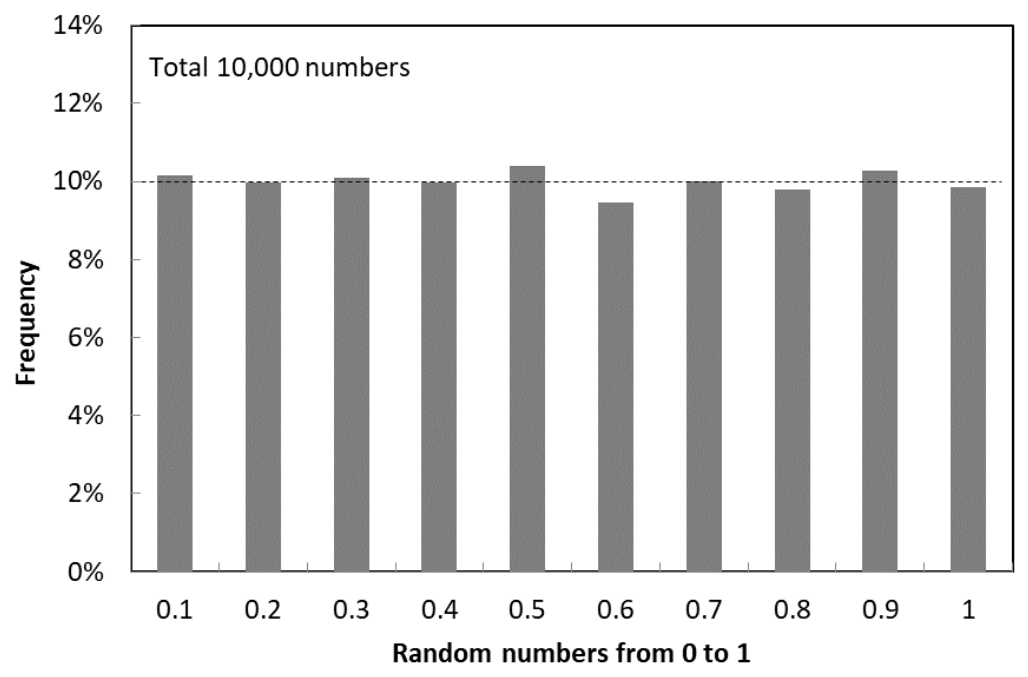

Figure 3. Distribution of random number sampling by Python's random.uniform function. 10,000 numbers were drawn, and the bin size of the histogram is 0.1 .

\section{Initial Energy and Free Space Lifetime}

The lifetime of excited particles in plasma gas is limited. At each collision, the excited molecule loses a certain amount of energy. Eventually, its lifetime of excited state ends, and it becomes a molecule with ground state energy when all the excited energy is lost (Fridman, 2008). However, the exact data about the initial energy, the energy lost by each collision, and the lifetime of the excited molecule is not available.

The current theoretical study employs the following approach. All excited molecules have the same initial energy $E_{0}$ (dimensionless number) at $t=0$. When a collision with another molecule occurs, the collision energy $E_{c}$, defined as the energy lost by each intermolecular collision, is subtracted from the molecule's energy $E$. The collision energy $E_{c}$ is set to 1 for a molecular collision. When the excited molecule collides with the wall or target microorganisms on the wall, it is assumed to lose more energy than the intermolecular collision. The exact impact on the energy is a function of wall or target materials and not known exactly. The wall collision energy $E_{w}$, defined as the energy lost by molecule-wall collision, is set to 10 in the current study. If there are no wall collisions or if there is no energy lost through wall collisions, the lifetime of the excited molecules is equivalent to the free space lifetime. When the excited molecule loses energy by wall collision more than the molecular collision, the molecule is deactivated at a faster rate in a confined geometry due to an increased frequency of wall collision.

The excited molecule with initial energy $E_{0}$ continuously loses its energy $E$ by successive collision with other molecules $\left(E_{c}\right)$ and wall $\left(E_{w}\right)$, and finally becomes a ground state molecule when $E$ becomes 0 . Consequently, the lifetime of the excited $\mathrm{N}_{2}$ molecules in a lumen is a function of gas temperature, gas pressure, molecule's initial energy, and geometry. The free space lifetime $t_{f l}$ of the excited molecules is defined as the time to lose all energies only through intermolecular collisions in free space without walls and obstacles. The free space lifetime is the key parameter that affects the degree of propagation in the lumen geometry.

Free space lifetime: $t_{f l}=E_{0} \times t_{s}=E_{0} / f$ 


\section{Results and Discussion}

\section{Calculation Conditions and Validation}

The $\mathrm{N}_{2}$ gas properties are calculated at each temperature and pressure by utilizing the gas kinetic theory, and each molecule's movement is investigated by the Monte Carlo method as explained above. Table 1 summarizes the operating condition used in the current study and the kinetic properties of $\mathrm{N}_{2}$ molecules. In terms of geometry, the narrow lumen is approximated as a tube with a square cross-section whose side is $2 \mathrm{~mm}$, and the length of the lumen is not confined in the current study to identify the propagation length of excited molecules. The ideal temperature of lowtemperature sterilization is below $60^{\circ} \mathrm{C}$ (Rutala et al., 2008). The current study adopts $55^{\circ} \mathrm{C}$ as the operating gas temperature.

The density of excited inert gas molecules to inactivate the microorganisms is a function of their lifetime that is dependent on the frequency of molecule and wall collisions. The exact information about the excited molecules' lifetime is not available for the current simulation conditions. The free space lifetime of the excited molecules is defined as a function of the allowable number of molecular collisions in free space. By combining Equations (1) and (3), the mean free path is inversely proportional to the pressure, and the collision frequency increases with pressure according to Equation (4). When the plasma is operated at atmospheric pressure (1 atm or 101,325 Pa), the lifetime of the excited molecule is very short due to too frequent collisions. Thus, it is preferable to run the system at a lower pressure. In the current simulation study, the operating pressures are $0.01 \mathrm{~atm}$ (or 1013.25 $\mathrm{Pa}$ ) and $0.001 \mathrm{~atm}$ (101.325 $\mathrm{Pa}$ ). As shown in Table 1, the free space lifetime depends on the pressure. With the identical amount of initial energy, the lifetime is increased 10 times when the pressure is decreased by 10 times.

Table 1. Operating condition and important kinetic properties of nitrogen molecule

\begin{tabular}{|l|l|l|}
\hline Lumen geometry: $L(\mathrm{~m})$ & 0.002 & $101.325(0.001 \mathrm{~atm})$ \\
\hline Temperature $\left({ }^{\circ} \mathrm{C}\right)$ & 55 & $2.24 \times 10^{22}$ \\
\hline Pressure $(\mathrm{Pa})$ & $1013.25(0.01 \mathrm{~atm})$ & 540.6 \\
\hline Number density $n\left(\mathrm{~m}^{-3}\right)$ & $2.24 \times 10^{23}$ & $7.60 \times 10^{-5}$ \\
\hline Average velocity $U(\mathrm{~m} / \mathrm{s})$ & 540.6 & $7.12 \times 10^{6}$ \\
\hline Mean free path $M F P(\mathrm{~m})$ & $7.60 \times 10^{-6}$ & $1.41 \times 10^{-7}$ \\
\hline Collision frequency $f(\mathrm{~Hz})$ & $7.12 \times 10^{7}$ & 10 \\
\hline Collision interval $t_{s}(\mathrm{~s})$ & $1.41 \times 10^{-8}$ & \\
\hline $\begin{array}{l}\text { Free space lifetime }(\mathrm{s}) \\
E_{0}=71,163,132\end{array}$ & 1 & \\
\hline
\end{tabular}



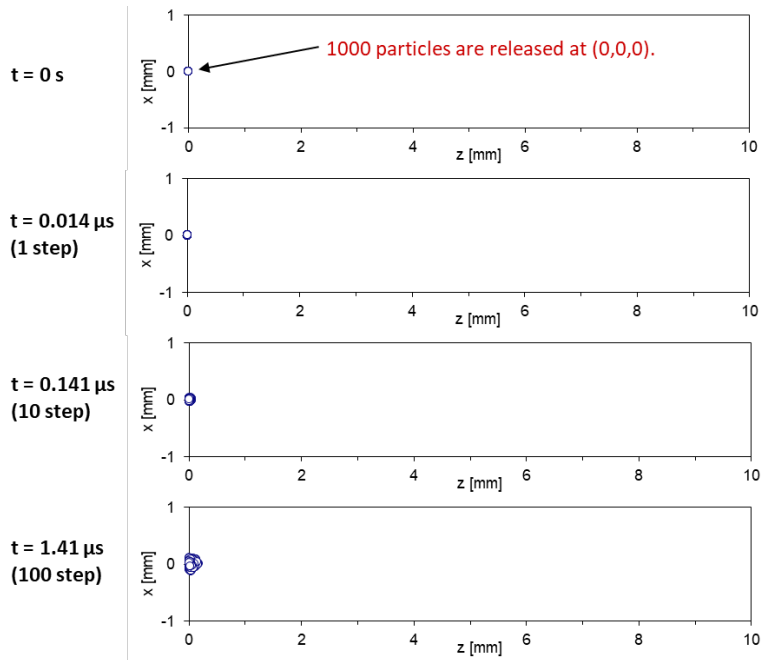
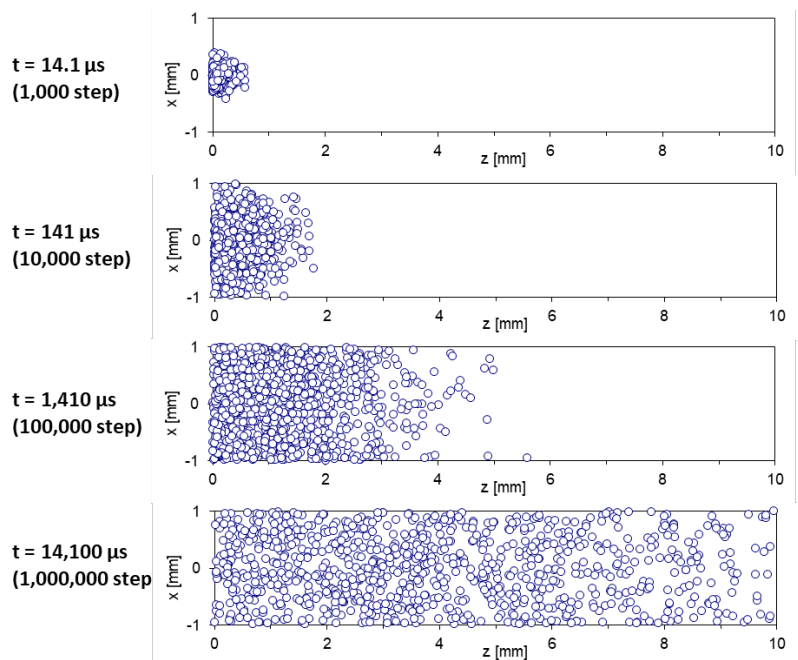

Figure 4. Positions of molecules at steps $0,1,10,100,1,000,10,000,100,000$, and 1,000,000. Each step is $0.0141 \mu \mathrm{s}$ and represents one intermolecular collision. Initially, 1000 molecules are released at the origin $(0,0,0)$. The molecules spread out by random molecular motions and collisions.

Figure 4. illustrates the propagation of excited molecules in the lumen with $2 \mathrm{~mm}$ width at 0.01 atm and $55^{\circ} \mathrm{C}$. Initially (at time $\left.t=0\right), 1,000$ excited molecules are released at the origin $(0,0,0)$. At each step, each molecule travels the mean free path MFP with the average velocity $U$ in a random direction to collide with other $\mathrm{N}_{2}$ molecules. In this figure, it is assumed that the excited molecules do not lose energy during collisions and continue to move with the given time frame. As time flows, the molecules, initially packed in a small volume near the origin, spread out by random molecular motion known as diffusion.

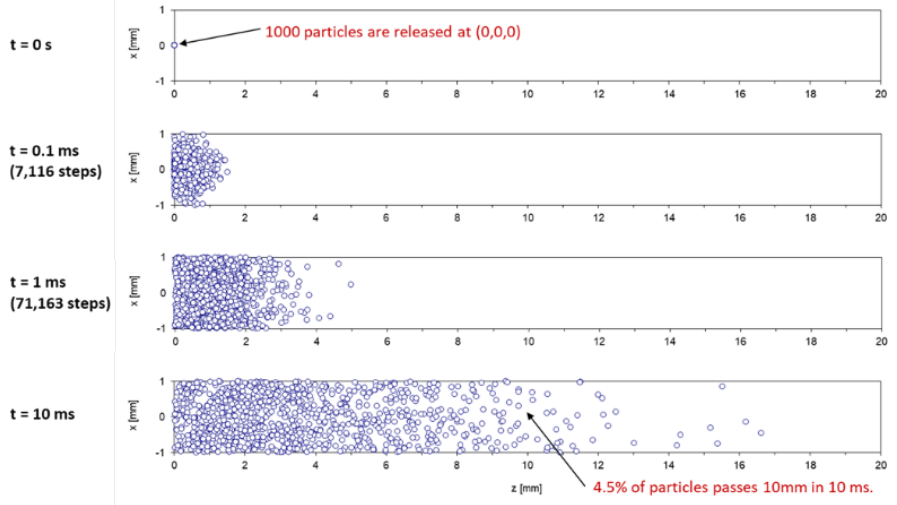

(a) Illustration of $\mathrm{N}_{2}$ molecules' location in the lumen

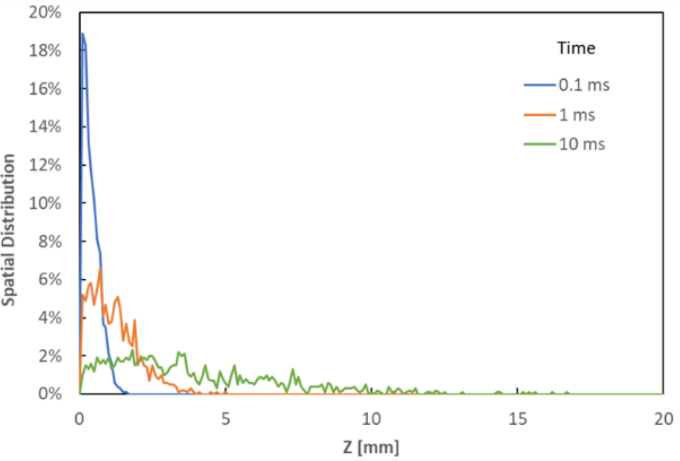

(b) Spatial distribution of $\mathrm{N}_{2}$ molecules at each time step

Figure 5. Distribution of molecules for time $t=0,0.1,1$, and $10 \mathrm{~ms}$. Initially $(t=0), 1,000$ molecules are released at the origin $(0,0,0) .1 \mathrm{~ms}$ represents 71,163 molecular collisions. In $10 \mathrm{~ms}, 4.5 \%$ of molecules propagate to $10 \mathrm{~mm}$ from the origin. The bin size of the histogram (b) is $0.1 \mathrm{~mm}$.

Figure 5. describes the distribution of 1,000 molecules at time $0.1,1$, and $10 \mathrm{~ms}$. $1 \mathrm{~ms}$ represents 71,163 collisions or steps. Figure 5(a) illustrates the molecules' locations at each time, and Figure 5(b) is the histogram showing the spatial distribution of molecules in the $z$-direction (along the length of the lumen) with the bin interval $0.1 \mathrm{~mm}$. Like the cases in Figure 4, it is assumed that the excited molecules do not lose energy during collisions. As time flows, 
the molecules, initially packed in a small volume near the origin, propagate and spread out as shown in Figure 5(a) and (b). From the simulation results presented in Figures 4 and 5, it is determined that the current simulation method can represent the molecule's propagation by random motion in the lumen and is applied to calculate the propagation and density of excited molecules.

\section{Propagation of Excited Molecules in a Lumen}

Figure 6 illustrates the molecules' life for the case the free space lifetime is $0.5 \mathrm{~s}$ at $0.01 \mathrm{~atm}$, and the wall collision energy is 10 . Initially (at time $t=0), 1,000$ excited molecules are released at the origin $(0,0,0)$ to simulate injection of the plasma gas into the lumen device. 1,000 molecules are a representative sample group used to calculate propagation and survival. The exact initial number or density of excited molecules is a function of the plasma generation method and its energy consumption (Fridman, 2008). Figure 6(a), a histogram with a bin size of $1 \mathrm{~mm}$, shows the position of each molecule when it loses its excited energy and its lifetime ends. It also represents the spatial decay frequency $\left(\mathrm{mm}^{-1}\right)$ defined as the number of molecules extinct at each location. The spatial decay ratio and survival ratios, depicted in Figures 6(b) and 6(c), are defined as the ratio of accumulated decayed molecules and survived molecules at each location to the total number of molecules, respectively. The survival ratio represents the density of excited molecules that inactivate microorganisms at each position. The survival ratio exponentially decreases along the length of lumen. The propagation length $l_{c}$ of the excited molecules is defined as the characteristic distance where $\mathrm{e}^{-1}(36.8 \%)$ of initially released molecules survive. This definition follows the concept of the penetration depth for radiation propagation inside the material. The propagation length is $28 \mathrm{~mm}$ for the current case. 


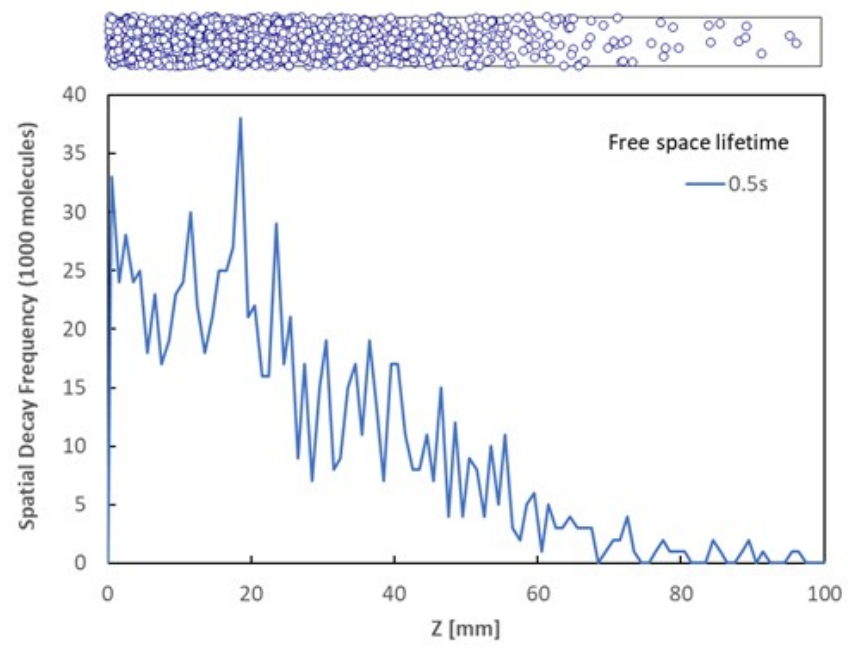

(a) End location of excited $\mathrm{N}_{2}$ molecules when the excite energy is lost and corresponding histogram showing the number of molecules extinct at each location. Bin size $=1 \mathrm{~mm}$

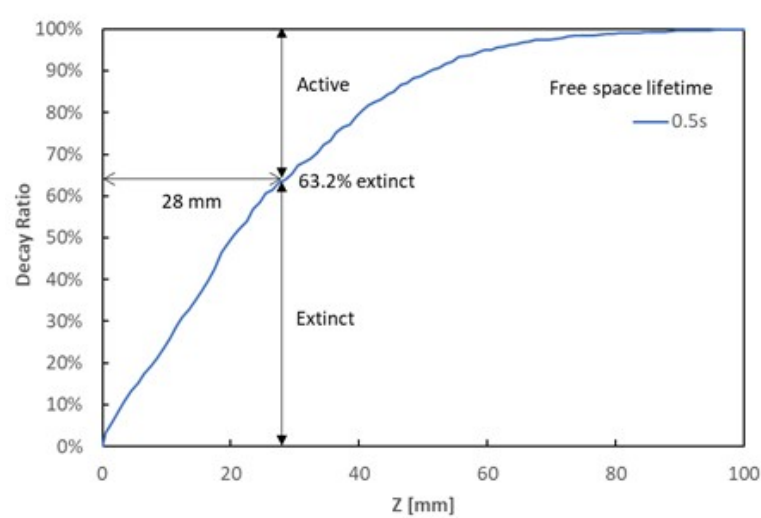

(b) Decay ratio and propagation length

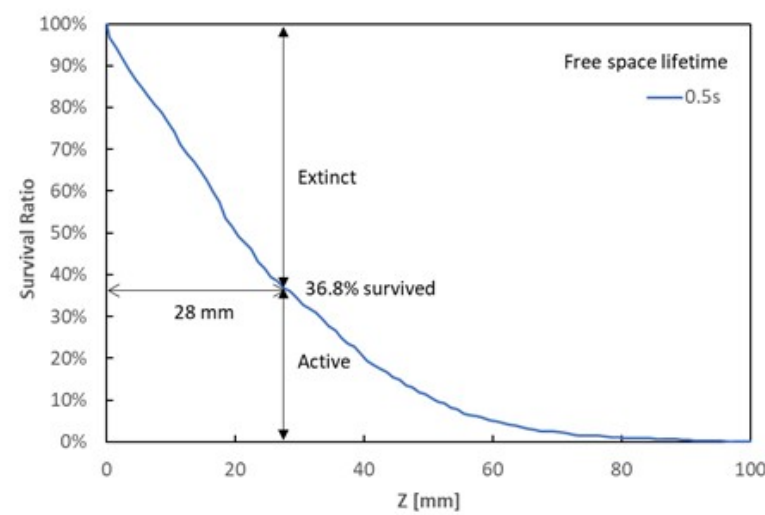

(c) Survival ratio and propagation length

Figure 6. Propagation of excited $\mathrm{N}_{2}$ molecules in a lumen at $0.01 \mathrm{~atm}$. 1,000 molecules are released at the origin $(0,0,0)$ at $t=0$. The free space lifetime is $0.5 \mathrm{~s}$ at $0.01 \mathrm{~atm}$. The propagation length is $28 \mathrm{~mm}$.

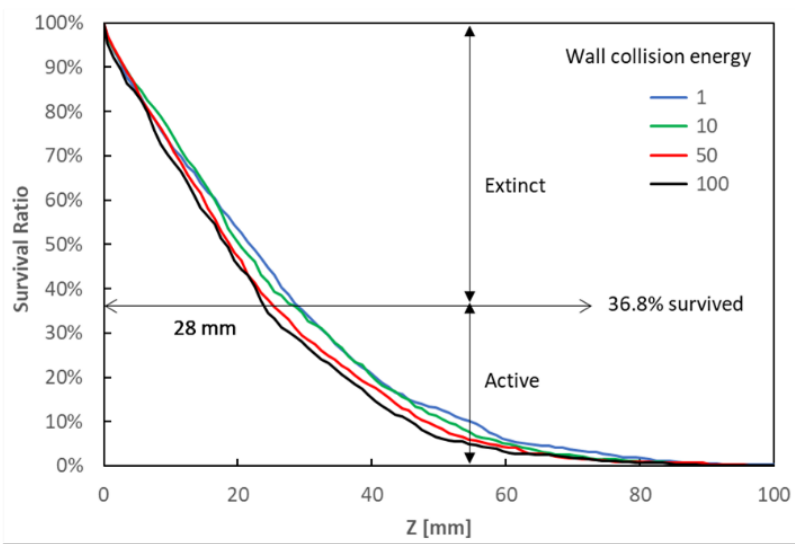

(a) Survival ratio along the lumen

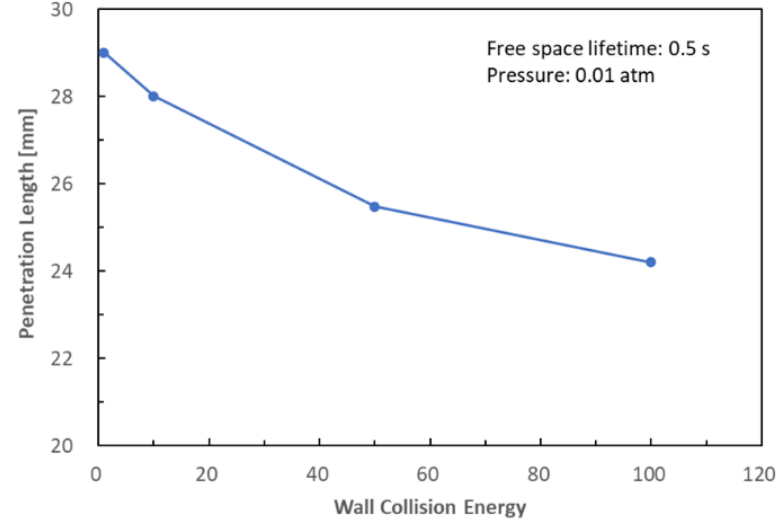

(b) Propagation length vs wall collision energy 
Figure 7. Survival ratio along the lumen and propagation length for various wall collision energies. The free space lifetime is $0.5 \mathrm{~s}$ at 0.01 atm. 1,000 molecules are released at the origin $(0,0,0)$ at $t=0$.

Figure 7. shows the survival ratio along the lumen length with various wall collision energy ranging from 1 to 100 while the free space lifetime is fixed at $0.5 \mathrm{~s}$ at $0.01 \mathrm{~atm}$. With the current geometry and operating condition, the effect of wall collision energy is not substantial. However, the simulation result indicates the exponential decrease of the propagation length when the wall collision energy is increases. As the energy loss by the wall collision increases, the excited molecules lose their energy faster and their propagation is limited. If the plasma gas is used for lumen sterilization, the wall material must be selected to minimize the energy loss of the excited particles by the wall collision. As mentioned above, we don't have references for the wall collision energy, it is assumed to be 10 in the following calculations.



Figure 8. Survival ratio along the lumen at 0.01 atm for various free space lifetimes $(0.01 \sim 1 \mathrm{~s})$. The initial energy $E_{0}$ ranges $711,631 \sim 71,163,123$. The propagation lengths are shown for $t_{f l}=0.1,0.5$, and $1 \mathrm{~s}$. The survival ratio represents the ratio of the number of available molecules at each position to the number of initially excited molecules.

Figure 8. shows the survival ratio along the lumen length at $0.01 \mathrm{~atm}$ at various free space lifetimes. As mentioned earlier, the free space lifetime is a function of pressure. $1 \mathrm{~s}$ of free space lifetime at 0.01 atm is equivalent to the initial molecular energy $E_{0}=71,163,132$ that can experience the same number of intermolecular collisions. The initial energy $E_{0}$ ranges $711,631 \sim 71,163,123$ for $0.01 \sim 1$ seconds of free space lifetime. The propagation lengths are $3.9,9.1,13.1,28.0,40.2 \mathrm{~mm}$ for $0.01,0.05,0.1,0.5,1$ seconds of free space lifetime, respectively. With $1 \mathrm{~s}$ of the free space lifetime at $0.01 \mathrm{~atm}\left(E_{0}=71,163,132\right)$, only $2.8 \%$ of excited molecules survive at $100 \mathrm{~mm}$ from the origin. The calculations indicate that the plasma at this pressure is ineffective for the sterilization of a long lumen.

The survival ratio along the narrow lumen at $0.001 \mathrm{~atm}$ is presented in Figure $9.10 \mathrm{~s}$ of the lifetime at 0.001 atm has the same initial energy $\left(E_{0}=71,163,132\right)$ as the case of $1 \mathrm{~s}$ of the lifetime at 0.01 atm because the collision frequency is inversely proportional to pressure. The propagation lengths are $107 \mathrm{~mm}$ for $t_{f l}=1 \mathrm{~s}$ and $369 \mathrm{~mm}$ for 10 s. When the free space lifetime is $1 \mathrm{~s}$ at $0.001 \mathrm{~atm}, 40 \%$ of excited molecules initially released at the origin are available for sterilization at $100 \mathrm{~mm}$. When the free space lifetime is $10 \mathrm{~s}$ at $0.001 \mathrm{~atm}, 82 \%$ of excited nitrogen molecules survive past $100 \mathrm{~mm}$. The survival or density at $100 \mathrm{~mm}$ in the lumen becomes 29 times higher when pressure decreases 10 times from 0.01 to 0.001 atm at the same initial excited energy level. If the same energy is applied to a unit amount of gas molecules, the initial density of excited particles at the origin decreases by 10 times when the pressure is decreased from 0.01 to 0.001 atm. However, the survival ratio increases by 29 times and thus the density of the 
excited molecules increases by 2.9 times from 0.01 atm to $0.001 \mathrm{~atm}$. It indicates that plasma at low pressure is highly effective in sterilizing or disinfecting narrow lumen devices.

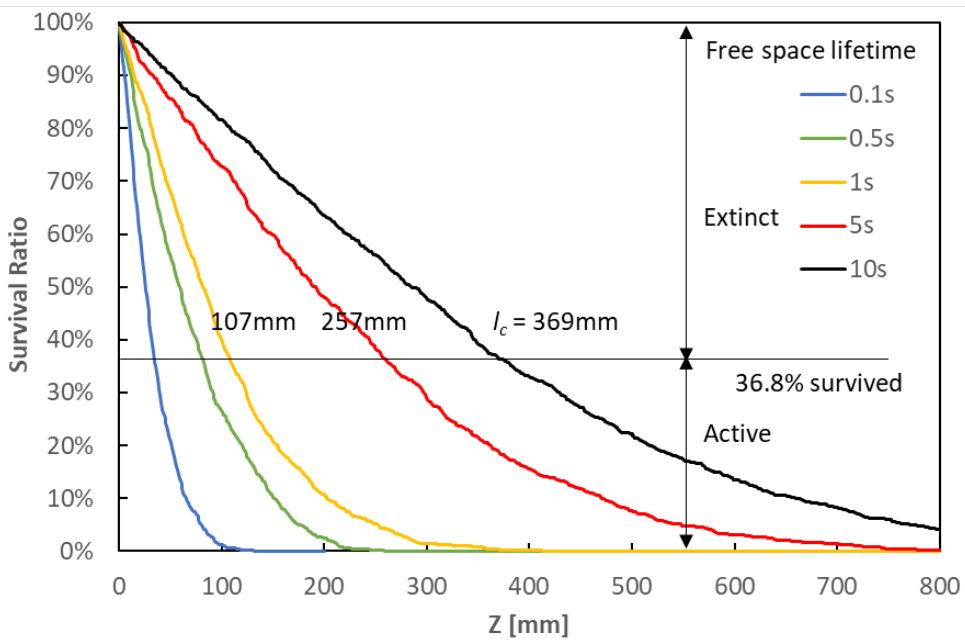

Figure 9. Survival ratio along the lumen at 0.001 atm for various free space lifetimes $(0.01 \sim 10 \mathrm{~s})$. The initial energy $E_{0}$ ranges $711,631 \sim 71,163,123$.

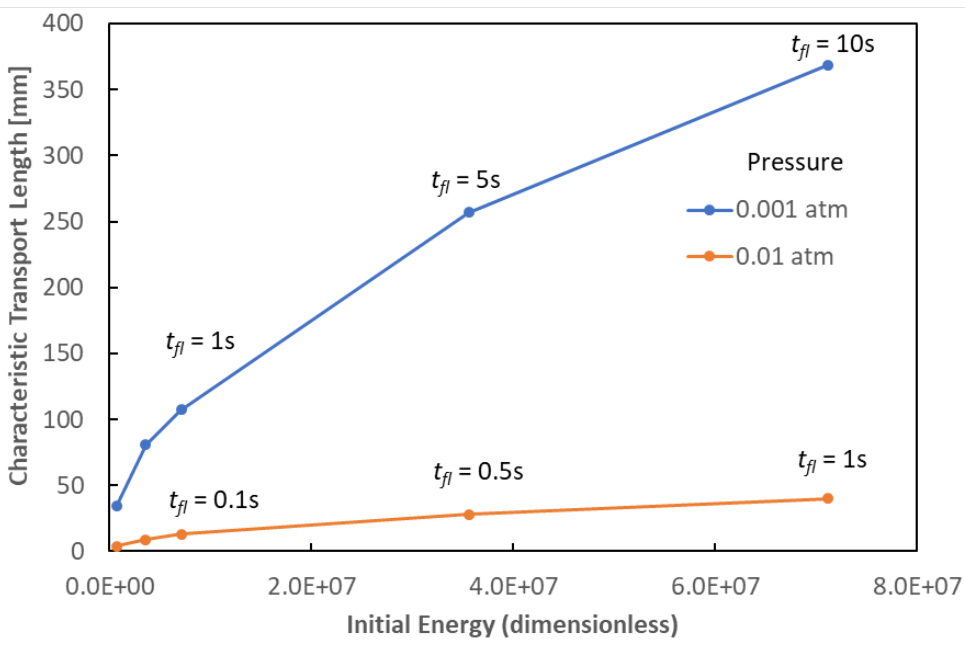

Figure 10. Propagation length for various initial energies. The free space lifetime is a function of initial energy and pressures. The propagation length at 0.001 atm is 9 times longer than that at $0.01 \mathrm{~atm}$.

Figure 10. depicts the propagation length as a function of the free space lifetime of excited molecules at 0.01 atm and $0.001 \mathrm{~atm}$. The free space lifetime is different at each pressure with the same initial energy $E_{0}$. When the free space lifetime is $10 \mathrm{~s}$ at $0.001 \mathrm{~atm}$, the propagation length is $369 \mathrm{~mm}, 185$ times the width of the lumen. At lower pressure, the excited molecules generated by plasma can travel further, and the lumen has a higher excited molecule density along its length, increasing plasma effectiveness for sterilization.

\section{Conclusion}

To understand the effectiveness of the plasma sterilization on narrow lumen medical devices, the Monte Carlo method was applied to predict the excited nitrogen molecule's propagation through random motion in a small lumen. The gas 
properties were obtained using the gas kinetic theory. In the current simulation approach, various values are assumed for free space lifetime or initial energy of excited nitrogen molecules, and a fixed value is assumed for wall collision.

In a lumen with a width of $2 \mathrm{~mm}$, it takes about $10 \mathrm{~ms}$ for $3.5 \%$ of $\mathrm{N}_{2}$ molecules to travel $10 \mathrm{~mm}$ at $55^{\circ} \mathrm{C}$ and $0.01 \mathrm{~atm}$. The simulation results strongly depend on the free space lifetime and initial energy level. The simulation result indicates that the survival ratio at $100 \mathrm{~mm}$ from the lumen opening becomes 29 times higher and the propagation length is 9 times longer when the pressure is decreased from $0.01 \mathrm{~atm}$ to $0.001 \mathrm{~atm}$. The increase in survival ratio is higher than the decrease in initial density of excited molecules due to the pressure decrease. It is strongly recommended to operate the plasma at a low pressure to sterilize the narrow lumen devices.

In a confined geometry, the plasma is unable to perform some of its inactivation mechanisms, like UV. However, plasma has multiple inactivation mechanisms, and the remaining mechanisms work with geometrical restriction and without material compatibility issues. The Monte Carlo method in the current study can be further developed with a more sophisticated molecule model, wall collision model, and plasma property measurement.

\section{Acknowledgments}

I would like to extend special thanks to Tarn Wilson and Andy Poggio for guiding me through the incredible process of learning and exploring the field of research and for supporting my growth as a student this past school year. And especially thank you to my dad Jaemo Koo for his support throughout my research career.

\section{References}

Brownlee, J. (2019, November 4). A Gentle Introduction to Monte Carlo Sampling for Probability. Website. https://machinelearningmastery.com/monte-carlo-sampling-for-probability/

Fridman, A. (2008). Plasma Chemistry. Cambridge University Press. https://doi.org/10.1017/CBO9780511546075

Harrison, R.L. (2010). Introduction To Monte Carlo Simulation. AIP Conference Proceedings, 1204(1), 17. https://doi.org/10.1063/1.3295638

Ismail, A.F., Khulbe, K., \& Matsuura, T. (2015). Gas Separation Membranes: Polymeric and Inorganic. Springer.

Kay, M \& Wyllie, R. (2011). Esophagogastroduodenoscopy and Related Techniques. Pediatric Gastrointestinal and Liver Disease, 4th edition, 626-649, https://doi.org/10.1016/B978-1-4377-0774-8.10061-2

Nandkumar, N. (2014). Plasma-The fourth state of matter. International Journal of Scientific \& Technology Research, 3(9), 49-52.

Prust, J. (2018, October). Sterilization of flexible endoscopes. Website. https://cdn.hpnonline.com/ce/pdfs/1810CEU.pdf

Rutala, W.A., Weber, D.J., \& HICPAC (Healthcare Infection Control Practices Advisory Committee). (2008). Guideline for Disinfection and Sterilization in Healthcare Facilities, Website. https://www.cdc.gov/infectioncon$\underline{\text { trol/guidelines/disinfection/ }}$

Sakudo, A., Yagyu, Y., \& Onodera, T. (2019). Disinfection and Sterilization Using Plasma Technology: Fundamentals and Future Perspectives for Biological Applications. International Journal of Molecular Sciences, 20(20), 5216. https://doi.org/10.3390/ijms20205216 
Sanchez, V. (2019). Sterilization of Flexible Endoscopes: Benefits \& Challenges. Website. https://www.censis.com/blog/sterilization-flexible-endoscopes-benefits-challenges/

Shintani, H., Sakuko, A., Burke, P., \& McDonnell, G. (2010). Gas plasma sterilization of microorganisms and mechanisms of action (Review), Experimental and Therapeutic Medicine, 1(5), 731-738.

https://doi.org/10.3892/etm.2010.136

Silva, J.M.F, Moreira, A. J., Bonato, C.B., Souza, S.B.K., Kikuchi, I.S., Ordonez, N., Mansano, R.D., \& Pinto, T.J.A. (2010). Pharmaceutical innovations in plasma sterilization technology. Industrial Pharmacy, $25,7-10$. https://www.researchgate.net/publication/242331031 Pharmaceutical innovations in pasma sterilization technology

Stapelmann, K., Fiebrandt, M., Raguse, M., Awakowicz, P., Reitz, G., \& Moeller, R. (2013). Utilization of lowpressure plasma to inactivate bacterial spores on stainless steel screws. Astrobiology, 13(7), 597-606.

https://doi.org/10.1089/ast.2012.0949

Traba, C., Chen, L., \& Liang, J.F. (2013). Low power gas discharge plasma mediated inactivation and removal of biofilms formed on biomaterials. Current Applied Physics, 13, S12-S18. https://doi.org/10.1016/j.cap.2012.12.024

Vincenti, W. G. \& Kruger, C. H. (1975). Introduction to Physical Gas Dynamics. Krieger Pub Co. 